\title{
LIE AND JORDAN STRUCTURE IN PRIME RINGS WITH DERIVATIONS
}

\author{
RAM AWTAR ${ }^{1}$
}

\begin{abstract}
In this paper Lie ideals and Jordan ideals of a prime ring $R$ together with derivations on $R$ are studied. The following results are proved: Let $R$ be a prime ring, $U$ be a Lie ideal or a Jordan ideal of $R$ and $d$ be a nonzero derivation of $R$ such that $u d(u)-d(u) u$ is central in $R$ for all $u$ in $U$. (i) If the characteristic of $R$ is different from 2 and 3 , then $U$ is central in $R$. (ii) If $R$ has characteristic 3 and $U$ is a Jordan ideal then $U$ is central in $R$; further, if $U$ is a Lie ideal with $u^{2} \in U$ for all $u$ in $U$, then $U$ is central in $R$. The case when $R$ has characteristic 2 is also studied. These results extend some due to Posner [2].
\end{abstract}

1. Introduction. A theorem of Posner [2] states that if $R$ is a prime ring, and $d$ is a nonzero derivation of $R$ such that, for all $r \in R, r d(r)-$ $d(r) r$ is in the centre of $R$, then $R$ is commutative. Our object is to generalize this theorem to Lie and Jordan ideals of $R$.

All rings considered here are associative. Let $R$ be a ring and $Z$ be its centre. For $x, y \in R,[x, y]=x y-y x$. For $a \in R$, let $I_{a}$ denote the inner derivation of $R$ by $a$; i.e., $I_{a}(x)=a x-x a$ for all $x \in R$. Throughout the paper $d$ denotes a nonzero derivation of $R$. For definitions see [1].

2. Basic lemmas. We begin with some preliminary lemmas.

LEMMA 1. If $R$ is a prime ring of characteristic different from 2 and $U$ is a Lie ideal of $R$ such that for all $u \in U,[u, d(u)] \in Z$, and $u^{2} \in U$, then $[u, d(u)]=0$ for all $u \in U$.

Proof. First observe that linearizing the relation $[u, d(u)] \in Z$ on $u=u+u^{2}$, we obtain $\left[u^{2}, d(u)\right]+[u, u d(u)+d(u) u] \in Z$. That is, $4[u, d(u)] u \in$ $Z$ for all $u \in U$. Hence, $[u, d(u)][u, r]=0$ for all $u \in U, r \in R$. If for some $u$ in $U,[d(u), u] \neq 0$, then, as it is in the centre $Z$, we get $[u, r]=0$ for all $r \in R$, in particular $[u, d(u)]=0$. Hence $[u, d(u)]=0$ for all $u \in U$.

Presented to the Society, October 10, 1972 under the title Lie structures and derivations in prime rings; received by the editors March 27, 1972 and, in revised form, February 20, 1973.

AMS (MOS) subject classifications (1970). Primary 16A66, 16A72; Secondary 16A48.

Key words and phrases. Prime rings, Lie ideal, Jordan ideal, derivation and inner derivation.

${ }^{1}$ This research was supported by the grant of C.S.I.R., India.

(c) American Mathematical Society 1973 
Lemma 2. Let $R$ be a prime ring and $U$ a Lie ideal of $R$. Suppose that $[u, d(u)] \in Z$ for all $u \in U$. Then $[[d(r), u], u] \in Z$ for all $u \in U, r \in R$. Further, if for all $u \in U,[u, d(u)]=0$ then $[[d(r), u], u]=0$ for all $r \in R$, $u \in U$.

Proof. Let $u \in U$ and $r \in R$, then $[u, r] \in U$, so that $[u+[u, r]$, $d(u+[u, r])] \in Z$. That is, $[[u, r], d(u)]+[u,[d(u), r]]+[u,[u, d(r)]] \in Z$. Now, $[[u, r], d(u)]+[u,[d(u), r]]=[r,[d(u), u]]$ for any $r \in R, u \in U$. Since $[d(u), u] \in Z$, we get $[[u, r], d(u)]+[u,[d(u), r]]=0$. Hence

$$
[[d(r), u], u] \in Z \text { for all } r \in R, u \in U \text {. }
$$

The last part can be obtained similarly.

The following lemma may have some independent interest.

LEMma 3. Let $R$ be a prime of characteristic not 2 and let $U$ be a Jordan ideal of $R$ with $u d(u)=d(u) u=0$ for all $u \in U$. Then $U=0$.

Proof. Linearize the relation $u d(u)=0$ on $u$ to get

$$
u d(v)+v d(u)=0 \text { for all } u, v \in U .
$$

For $u \in U$ and any $r \in R, u(u r-r u)+(u r-r u) u \in U$. But $2\left(r u^{2}-u^{2} r\right)=$ $\{u(r u-u r)+(r u-u r) u\}-\{(u r-r u) u+u(u r-r \dot{u})\}$. As the first and second term on the right hand side are in $U, 2\left(r u^{2}-u^{2} r\right) \in U$. As $2 u^{2} \in U$, $2\left(u^{2} r+r u^{2}\right) \in U$. It follows that $4 u^{2} r$ and $4 r u^{2}$ are in $U$. Replacing $v$ by $4 r u^{2}$ where $r \in R$ in (1) and using the hypothesis, we get $u d(r) u^{2}=0$ for all $u \in U, r \in R$. If in (1) we replace $v$ by $u r+r u$ where $r \in R$, then $u^{2} d(r)+$ $u d(r) u+2 u r d(u)=0$; and hence $u^{2} d(r) u+u d(r) u^{2}=0$. Therefore, $u^{2} d(r) u=$ 0 for all $u \in U$ and $r \in R$. Again, put $v=4 u r u=2\{u(u r+r u)+(u r+r u) u\}-$ $\left\{2 u^{2} \cdot r+r \cdot 2 u^{2}\right\}$ in (1) where $r \in R$; then $0=u d(u) r u+u^{2} d(r) u+u^{2} r d(u)=$ $u^{2} d(r) u+u^{2} r d(u)$. Hence, $u^{2} r d(u)=0$ for all $r \in R, u \in U$. Lastly, replace $v$ by $4 u^{2} r$ in (1), for $r \in R$; then $0=u d\left(4 u^{2} r\right)+4 u^{2} r d(u)=4 u^{3} d(r)$. Hence, $u^{3} d(r)=0$ for all $u \in U$ and $r \in R$. Then by Lemma 1 of [2], $u^{3}=0$ for all $u \in U$. For $u \in U$ and $r \in R, 2\left(u^{2} r+r u^{2}\right) \in U$, so that $0=2^{3}\left(u^{2} r+r u^{2}\right)^{3}$. Multiply on the right by $u^{2} r$, to obtain $2^{3}\left(u^{2} r\right)^{4}=0$. Hence, $\left(u^{2} r\right)^{4}=0$. If for some $u$ in $U, u^{2} \neq 0$, then $u^{2} R$ is a nonzero right ideal of $R$ in which the quartic of every element is zero. By Levitzki's theorem [1, Lemma 1.1] $R$ would have a nilpotent ideal; which is impossible for a prime ring. Hence $u^{2}=0$ for all $u \in U$. By repeating the above argument we can show that $u=0$ for all $u \in U$.

\section{The main theorems.}

THEOREM 1. Let $R$ be a prime ring of characteristic different from 2 and 3. Let $d$ be a nonzero derivation of $R$, and $U$ a Lie ideal of $R$ with $[u, d(u)] \in Z$ for all $u$ in $U$. Then $U \subset Z$. 
Proof. By Lemma 2, $[[d(r), u], u] \in Z$ for all $u \in U, r \in R$. Now, proceeding on the same lines as in Posner [2] (cf. equations (16) to (27)), we have $[d(u), u]=0$ for all $u \in U$. Again by Lemma 2 ,

$$
[[d(r), u], u]=0 \text { for all } u \in U, r \in R .
$$

Replace $u$ by $u+w$ with $w \in U$ in (2).

$$
[[d(r), u], w]+[[d(r), w], u]=0 \text { for all } r \in R, u, w \in U .
$$

Suppose now that $w, v \in U$ are such that $w v$ is also in $U$. By replacing $w$ by $w v$ in (3), where $v \in U$, and expanding we get

$$
\begin{aligned}
w[[d(r), u], v]+ & {[[d(r), u], w] v+[d(r), w][v, u] } \\
+ & {[[d(r), w], u] v+w[[d(r), v], u]+[w, u][d(r), v]=0 . }
\end{aligned}
$$

In view of (3) the last equation reduces to $[d(r), w][v, u]+[w, u][d(r), v]=$ 0 . For any $t \in R, w \in U$, the element $v=t w-w t$ satisfies the criterion $w v \in U$, hence by above

(4) $[d(r), w][[t, w], u]+[w, u][d(r),[t, w]]=0$ for $t, r \in R ; u, w \in U$.

Putting $u=w$ in (4), we have

$$
[d(r), w][[t, w], w]=0 \quad \text { for } r, t \in R \text { and } w \in W .
$$

Substitution of $t d(a)$ for $r$ in (5) with $a \in R$ yields on expansion

$$
[d(r), w]\{2[t, w][d(a), w]+[[t, w], w] d(a)+t[[d(a), w], w]\}=0 .
$$

By (5) the second term is zero and by (2) the third term is zero, so that

$$
[d(r), w][t, w][d(a), w]=0 \text { for all } r, t, a \in R, w \in U .
$$

Put $u=[t, w]$ in (4). Then $[[t, w], w][[t, w], d(r)]=0$. Its linearization on $t=t+d(a)$ where $a \in R$ together with (2) yields

$$
[[t, w], w][[d(a), w], d(r)]=0 \text { for all } a, t, r \in R \text { and } w \in U .
$$

Replace $t$ by $d(t) p$ with $p \in R$ in (7) and expand; then $\{2[d(t), w][p, w]+d(t)[[p, w], w]+[[d(t), w], w] p\}[[d(a), w], d(r)]=0$. By (7) the second term is zero, while by (2) the third term is zero. Hence

$$
[d(t), w][p, w][[d(a), w], d(r)]=0 .
$$

In view of (6), the last equation reduces to

$$
[d(t), w][p, w] d(r)[d(a), w]=0 \text { for all } a, r, p, t \in R \text { and } w \in U .
$$

In (6) replace $t$ by $\operatorname{td}(p)$, where $p \in R$ and using the last equation to get $[d(r), w] R[d(p), w][d(a), w]=0$ for all $r, p, a \in R$ and $w \in U$. 
Now, if $[d(r), w]=0$ for all $r \in R, w \in U$, that is for all $r \in R, w \in U$, $\left(I_{w} d\right) r=0$, then by [2, Theorem 1] $w \in Z$ for all $w \in U$. Thus assume that there exists a $w \in U$ such that for some $r \in R,[d(r), w] \neq 0$. That is $w \notin Z$. Then for all $a, p \in R$

$$
[d(p), w][d(a), w]=0 .^{2}
$$

Replace $a$ by $b c$ where $c, b \in R$ and expand to get

$$
\begin{aligned}
{[d(p), w][d(b), w] c } & +[d(p), w] d(b)[c, w] \\
& +[d(p), w] b[d(c), w]+[d(p), w][b, w] d(c)=0 .
\end{aligned}
$$

Replace $b$ by $[t, w]$ where $t \in R$. By (8) the first term is zero, while by (6) the third term is zero, and by (5) the fourth term is zero. Therefore,

$$
[d(p), w] d([t, w])[w, c]=0 .
$$

Since, $d([t, w])=[d(t), w]+[t, d(w)]$ and using (8) we get

$$
[d(p), w][t, d(w)][w, c]=0 \text { for all } p, c, t \in R \text { and } w \in U .
$$

Replace $c$ by $c r_{1}$ where $r_{1} \in R$, then $[d(p), w][t, d(w)] R[w, c]=0$. Since $R$ is prime and $w \notin Z$, we get $[d(p), w][t, d(w)]=0$ for $p, t \in R, w \in U$. Therefore, $[d(p), w] R[t, d(w)]=0$ for $p, t \in R$ and $w \in U$; which together with $[d(r), w] \neq 0$ implies that $d(w) \in Z$.

Now suppose that $u \in U$ and $u \in Z$. Then $0=d[u, a]=[d(u), a]+$ $[u, d(a)]$ and hence $d(u) \in Z$. Therefore, $d(u) \in Z$ for all $u \in U$, so that $d([w, a]) \in Z$ for all $a \in R$. That is, $[d(w), a]+[w, d(a)] \in Z$ for all $a \in R$. Thus $[w, d(a)] \in Z$ for all $a \in R$. In particular,

$$
\left[w^{\prime}, d(a w)\right]=[w, d(a)] w+[w, a] d(w) \in Z .
$$

Commute (9) with $w$ to get $[w,[w, a]] d(w)=0$ for $a \in R$. If $d(w) \neq 0$, and as it is in the centre $Z,[w,[w, a]]=0$ for all $a \in R$. By [1, Sublemma, p. 5] $w \in Z$, a contradiction. Hence, $d(w)=0$. Thus, by (9), $[w, d(a)] w \in Z$ for all $a \in R$; that is $[d(a), w][w, b]=0$ for $a, b \in R$. Replace $b$ by $b c$, where $c \in R$, then $[d(a), w] R[w, b]=0$. Since $R$ is prime, either $w \in Z$ or $[d(a), w]=0$ for all $a \in R$. So, in both cases $w \in Z$; a contradiction. Hence the conclusion is that $w \in Z$ for all $w \in U$. This proves the theorem.

Now we should like to settle the problem when $R$ has characteristic 3 . Note that the assumption that the characteristic is different from 3 does not enter the proof of Theorem 1 onwards of equation $[u, d(u)]=0$ for all $u \in U$. Therefore, if $[u, d(u)]=0$ holds for all $u \in U$, we can show that $U \subset Z$ even if $R$ has characteristic 3. In view of Lemma 1, if $R$ has characteristic different from 2 and $U$ is a Lie ideal of $R$ such that for all $u \in U$,

2 Onward proof of this theorem is given by the referee. 
$u^{2} \in U$ and $[u, d(u)] \in Z$, then $[u, d(u)]=0$ for all $u \in U$. Hence, we get the following weaker result.

THEOREM 2. Let $R$ be a prime ring of characteristic 3 and $d$ a nonzero derivation of $R$. If $U$ is a Lie ideal of $R$ with $[u, d(u)] \in Z$ and $u^{2} \in U$ for all $u \in U$, then $U \subset Z$.

Now we will show that the conclusion of Theorems 1 and 2 holds even if $U$ is a Jordan ideal of $R$. In this regard, we prove the following.

THEOREM 3. Let $R$ be a prime ring of characteristic not equal to 2. Let $d$ be a nonzero derivation of $R$ and $U$ be a Jordan ideal of $R$, such that $[u, d(u)] \in Z$ for all $u \in U$. Then $U \subset Z$.

Proof. For $u \in U, 2 u^{2} \in U$. Therefore by Lemma $1,[u, d(u)]=0$ for all $u \in U$. Replace $u$ by $u+v$, where $v \in U$, then

$$
[u, d(v)]+[v, d(u)]=0 \text { for all } u, v \in U .
$$

In (10), replace $v$ by $u r+r u, r \in R$, and expand to get

$$
\begin{aligned}
u[u, d(r)]+[u, d(r)] u & +d(u)[u, r] \\
& +[u, r] d(u)+u[r, d(u)]+[r, d(u)] u=0,
\end{aligned}
$$

i.e.,

(11) $2 u r d(u)-2 d(u) r u+u^{2} d(r)-d(r) u^{2}=0$ for $r \in R, u \in U$.

Replace $r$ by $u r$ in (11), then

$$
d(u)\left(u^{2} r-r u^{2}\right)=0 \text { for all } r \in R, u \in U
$$

that is, $d(u) I_{u^{2}}(r)=0$ for all $r \in R, u \in U$; hence by [2, Lemma 1], either

$$
u^{2} \in Z \quad \text { or } \quad d(u)=0 \text { for all } u \in U .
$$

For $u \in U$ and any $r \in R, u r+r u \in U$. But

$$
4 u r u=2\{u(u r+r u)+(u r+r u) u\}-\left\{2 u^{2} \cdot r+r \cdot 2 u^{2}\right\} .
$$

The first and the second term on the right are in $U$. Hence $4 u r u \in U$. Therefore, if we replace $v$ by 4uru in (10), where $r \in R$, then i.e.,

$$
d(u)[u, r] u+u[u, d(r)] u+u[u, r] d(u)+u[r, d(u)] u=0,
$$

(14) $u^{2} r d(u)-d(u) r u^{2}+u^{2} d(r) u-u d(r) u^{2}=0$ for $r \in R, u \in U$.

Replace $r$ by $u r$ in (14) and use (14) to get $u d(u)\left(u r u-r u^{2}\right)=0$. However in view of (12), this equation reduces to $u d(u) u(u r-r u)=0$. That is, $u d(u) u \cdot I_{u}(r)=0$. By [2, Lemma 1], either

$$
u d(u) u=0 \text { or } u \in Z \text { for all } u \in U \text {. }
$$


In (12), replace $u$ by $u+v$ where $v \in U$ and use (12). Then

$$
\{d(u)+d(v)\}[u v+v u, r]+d(u)\left[v^{2}, r\right]+d(v)\left[u^{2}, r\right]=0 .
$$

Replace $u$ by $-u$, then

$$
\{-d(u)+d(v)\}[-u v-v u, r]-d(u)\left[v^{2}, r\right]+d(v)\left[u^{2}, r\right]=0 .
$$

Adding last two equations and dividing by 2 , we have $d(u)[u v+v u, r]+$ $d(v)\left[u^{2}, r\right]=0$ for all $r \in R$ and $u, v \in U$. By Lemma 3, $u d(u) \neq 0$, for some $u$ in $U, d(u) \neq 0$, hence by (13) $u^{2} \in Z$. The net result of this is

$$
d(u)[u v+v u, r]=0 .
$$

That is, $d(u) I_{u v+v u}(r)=0$ for all $r \in R$ and $v \in U$. By [2, Lemma 1] $u v+v u \in$ $Z$ for all $v \in U$. If $u^{2}=0$, then $0=d\left(u^{2}\right)=u d(u)+d(u) u=2 u d(u)$ so that $u d(u)=0$, a contradiction. Hence $u^{2} \neq 0$. Suppose that $u d(u) u=0$ then $u^{2} d(u)=0$ which implies that $d(u)=0$, a contradiction. Hence $u d(u) u \neq$ 0 , so (15) gives $u \in Z$. Hence $2 u v \in Z$; so that $u v \in Z$ for all $v \in U$. As $u(\neq 0) \in Z$, we have $v \in Z$ for all $v \in U$. Hence $U \subset Z$. This completes the proof of Theorem 3 .

We should like to settle the problem even when $R$ has characteristic 2 . In this case Lie ideals and Jordan ideals will coincide. We are proving now the following weaker result.

THEOREM 4. Let $R$ be a prime ring of characteristic 2, and let $d$ be $a$ nonzero derivation of $R$. Let $U$ be a Lie (Jordan) ideal and a subring of $R$. Suppose that $[u, d(u)] \in Z$ for all $u \in U$. Then $U$ is commutative.

Proof. By Lemma $2,[[d(r), u], u] \in Z$ i.e.,

$$
d(r) u^{2}+u^{2} d(r) \in Z \text { for all } r \in R, u \in U .
$$

Commuting (16) with $d(r)$ and $u^{2}$ respectively, we get

$$
u^{2} d(r)^{2}=d(r)^{2} u^{2} \quad \text { for all } r \in R, u \in U
$$

and

$$
u^{4} d(r)=d(r) u^{4} \quad \text { for all } r \in R, u \in U
$$

where $d(r)^{2}$ stands for $(d(r))^{2}$.

In (17a) replace $r$ by $v+u^{2} v$ where $v \in U$ and use (17a). Then

$$
\begin{aligned}
& \left(u^{2} d(v)\right)^{2}+u^{2} d(v) d\left(u^{2}\right) v+u^{2} d\left(u^{2}\right) v d(v)+u^{4} d(v)^{2} \\
& =\left(d(v) u^{2}\right)^{2}+d(v) d\left(u^{2}\right) v u^{2}+d\left(u^{2}\right) v d(v) u^{2}+u^{2} d(v)^{2} u^{2} .
\end{aligned}
$$

For $u \in U, d\left(u^{2}\right)=u d(u)+d(u) u \in Z$, so that in view of (17b) the last equation reduces to $\left(u^{2} d(v)+d(v) u^{2}\right)^{2}=0$ for $u, v \in U$. Since $R$ is prime, 
by using (16) we get

$$
u^{2} d(v)=d(v) u^{2} \quad \text { for all } u, v \in U .
$$

Replace $u$ by $u+w$ where $w \in U$ in (18). Then

$$
(u w+w u) d(v)=d(v)(u w+w u) .
$$

Replace $w$ by $w u$, then $(u w+w u) u d(v)=d(v)(u w+w u) u=(u w+w u) d(v) u$. Therefore, $(u w+w u)(u d(v)+d(v) u)=0$ for all $u, v, w \in U$. Linearize the last equation on $u=u+u_{1}^{2}$, where $u_{1} \in U$ and put $v=u$. Then using (18) we get

$$
\left(u_{1}^{2} w+w u_{1}^{2}\right)(u d(u)+d(u) u)=0 \text { for all } u, v, w \in U .
$$

If $[d(u), u] \neq 0$ for some $u$ in $U$, then $u_{1}^{2} w=w u_{1}^{2}$ for all $u_{1}, w \in U$; so that, $u^{2}(w r+r w)=(w r+r w) u^{2}$ for all $r \in R, u, w \in U$. That is, $w\left(u^{2} r+r u^{2}\right)=$ $\left(u^{2} r+r u^{2}\right) w$ for all $r \in R, u, w \in U$. Replace $r$ by $r u$, then $\left(u^{2} r+r u^{2}\right) \times$ $(w u+u w)=0$ for all $r \in R, u, w \in U$. Replacing $w$ by $[u, t]$ we get

$$
\left(u^{2} r+r u^{2}\right)\left(u^{2} t+t u^{2}\right)=0 \text { for all } r, t \in R, u \in U .
$$

Replace $t$ by $t p$ where $p \in R$; then $\left(u^{2} r+r u^{2}\right) R\left(u^{2} t+t u^{2}\right)=0$. Since $R$ is prime, we get $u^{2} \in Z$ for all $u \in U$. Thus assume that $[d(u), u]=0$ for all $u \in U$. By Lemma 2, [[d(r),u],u]=0 i.e., $u^{2} d(r)=d(r) u^{2}$ for all $r \in R$, $u \in U$. Replace $r$ by $r a$ where $a \in R$, then

$$
d(r)\left(u^{2} a+a u^{2}\right)+\left(u^{2} r+r u^{2}\right) d(a)=0 .
$$

For $v \in U, d\left(v^{2}\right)=v d(v)+d(v) v=0$. Hence $d(r)\left(u^{2} v^{2}+v^{2} u^{2}\right)=0$ for all $r \in R, v \in U$. Thus by [2, Lemma 1] $u^{2} v^{2}=v^{2} u^{2}$ for $u, v \in U$. Therefore $u^{2}(v w+w v)=(v w+w v) u^{2}$ for $u, v, w \in U$. Replace $v$ by $v w$, then $(v w+w v) \times$ $\left(u^{2} w+w u^{2}\right)=0$; so that $\left(w^{2} r+r w^{2}\right)\left(u^{2} w+w u^{2}\right)$, i.e., $I_{w^{2}}(r)\left(u^{2} w+w u^{2}\right)=0$ for all $r \in R, u, w \in U$. The Lemma 1 of [2] forces that if $w^{2} \notin Z$ for some $w$ in $U$, then for that $w, u^{2} w=w u^{2}$ for all $u \in U$. So that, $[[u, v], w]=0$ for all $u, v \in U$. For $w \in U$, then $[[v, w], u]+[[w, u], v]=[[u, v], w]=0$ for all $u, v \in U$. Replace, in $[[v, w], u]+[[w, u], v]=0, v$ by $v w$ and expand to obtain $[[v, w], u] w+[v, w][w, u]+[[w, u], v] w=0$. Hence, $[v, w][w, u]=0$ for all $u, v \in U$. Replacing $v$ by $[w, r]$ and $u$ by $[w, t]$, we get

$$
\left(w^{2} r+r w^{2}\right)\left(w^{2} t+t w^{2}\right)=0 \text { for all } r, t \in R \text {. }
$$

Replace $t$ by $t p$ where $p \in R$, then $\left(w^{2} r+r w^{2}\right) R\left(w^{2} t+t w^{2}\right)=0$, which implies that $w^{2} \in Z$, a contradiction. Hence the conclusion is that $u^{2} \in Z$ for all $u \in U$. So in all possible cases $w^{2} \in Z$ for all $u \in U$ so that $(u v+v u) \in$ $Z$ and $(u v+v u) u \in Z$ for all $u, v \in U$. If $u \notin Z(U)$, where $Z(U)$ denotes the centre of $U$, then $u v+v u=0$ for all $v \in U$ and $u \in Z(U)$. Hence $U$ is commutative. 
In Theorem 4, if we just assume that $U$ is only a Lie (Jordan) ideal or only a subring of $R$, then $U$ may not be commutative. This is shown by the following examples.

EXAMPLE 1. Let $R$ be a prime ring of all $2 \times 2$ matrices over a noncommutative prime ring. Consider $U=\left\{\left(\begin{array}{cc}\alpha & 0 \\ 0 & \beta\end{array}\right) \in R\right\}$. It is clear that $U$ is a subring, but not a Lie ideal of $R$. Let us define $d: R \rightarrow R$ such that

$$
d\left(\begin{array}{ll}
\alpha & \beta \\
\gamma & \delta
\end{array}\right)=\left(\begin{array}{cc}
0 & -\beta \\
\gamma & 0
\end{array}\right), \quad \text { for all }\left(\begin{array}{ll}
\alpha & \beta \\
\gamma & \delta
\end{array}\right) \in R .
$$

It is easy to verify that $d$ is a nonzero derivation of $R$ with $[u, d(u)] \in Z$ for all $u \in U$. But $U$ is not commutative.

EXAMPLE 2. Consider the prime ring $R$ of all $2 \times 2$ matrices over $G F(2)$. Let $U=\left\{\left(\begin{array}{cc}a & b \\ c & a\end{array}\right), a, b, c \in G F(2)\right\}$. It is clear that $U$ is a Lie ideal but not a subring of $R$. Let us define $d: R \rightarrow R$ such that

$$
d\left(\begin{array}{ll}
a & b \\
c & d
\end{array}\right)=\left(\begin{array}{ll}
d-c & a-d \\
a-d & b-c
\end{array}\right) \text { for all }\left(\begin{array}{ll}
a & b \\
c & d
\end{array}\right) \in R .
$$

It can be seen that $d$ is a nonzero derivation of $R$ with $[u, d(u)] \in Z$ for all $u \in U$. However, $U$ is not commutative.

Following example shows that a ring may satisfy all the assumptions of Theorem 4, but $U$ may not be in the centre, even though $U$ is commutative.

EXAMPLE 3. Let $R$ be a ring of all $2 \times 2$ matrices with entries from $G F(2)$. Consider $U=\left\{\left(\begin{array}{ll}a & b \\ b & a\end{array}\right), a, b \in G F(2)\right\}$. It can easily be verified that $U$ is both a Lie (Jordan) ideal and a subring of $R$, but it is not an ideal of $R$. Define $d: R \rightarrow R$ as in Example 2. Then $d$ satisfies $[u, d(u)] \in Z$ for all $u \in U$. Clearly $U$ is commutative, but $U$ is not in the centre of $R$.

Author wishes to express his thanks to Dr. Surjeet Singh of Aligarh Muslim University for his continuous encouragement and suggestions during the preparation of this paper. He also wishes his thanks to the referee for his valuable suggestions, particularly in the proof of Theorem 1.

\section{REFERENCES}

1. I. N. Herstein, Topics in ring theory, Univ. of Chicago Press, Chicago, Ill., 1969. MR 42 \#6018.

2. E. C. Posner, Derivations in prime rings, Proc. Amer. Math. Soc. 8 (1957), 10931100. MR 20 \#2361.

Department of Mathematics and Statistics, Aligarh Muslim University, Aligarh, Uttar Pradesh, India 